\title{
Myocardial Ischemia due to a Type IV Dual LAD with the Long LAD Arising from the Right Sinus of Valsalva: A Case Report and Literature Review
}

\author{
Xiangyu Gao, Hongwei Li and Hui Chen
}

\begin{abstract}
We herein report the case of a 60-year-old man who presented with chest discomfort. Coronary angiography demonstrated that the short left anterior descending artery (LAD) arose from the left main coronary artery and the long LAD from the right coronary sinus of Valsalva, which was confirmed by 64-slice multidetector computed tomography coronary angiography. Single-photon emission computed tomography revealed a significant, almost irreversible degree of hypoperfusion in the anterior wall of the left ventricle, thus indicating that the short LAD was associated with myocardial ischemia and severe atherosclerotic lesions. Therefore, SPECT showed that the myocardium was largely necrotic, and we did not perform angioplasty for the short LAD.
\end{abstract}

Key words: double left anterior descending artery, coronary artery anomaly, computed tomography angiography, coronary angiography, SPECT, myocardial ischemia

(Intern Med 54: 2619-2623, 2015)

(DOI: 10.2169/internalmedicine.54.4147)

\section{Introduction}

Most coronary artery anomalies are found incidentally by coronary angiography or multidetector-row computed tomography (MDCT). The dual left anterior descending artery (LAD) is a rare coronary artery anomaly, classified into one of six types according to the origin and course of the long LAD (1). Spindola-Franco et al. (2) published an angiographic description of dual LAD with four subtypes. In types I-III, both the short LAD and the long LAD originate from the left main coronary artery (LMCA) or the proximal portion of the LAD. Type IV is a short LAD terminating in the mid-portion of the anterior interventricular sulcus and a long LAD originating from the right coronary artery (RCA) and reaching to the anterior interventricular sulcus. Type V was proposed in 2010 by Manchanda et al. (3), in which a short LAD originates independently from the left sinus of Valsalva, and the long LAD originates from the right sinus of Valsalva (RSV) with an intramyocardial course before reaching the distal interventricular groove. Type VI was de- fined in 2011 by Maroney and Klein (4). Type VI refers to the short LAD that originates from the LMCA, with the long LAD arising from the RCA and following a route underneath the right ventricular outflow tract to the anterior interventricular groove.

We herein report a rare case of anomalous origin of a long LAD coronary artery arising from the RSV and a short LAD arising from the LMCA. The anomalous origin of a coronary artery does not generally lead to myocardial ischemia, but the present patient had significant hypoperfusion in a portion of the anterior wall of the left ventricle and anterior septal myocardium.

\section{Case Report}

A 60-year-old man was admitted to our department for atypical chest discomfort without inducement, which he had complained of for the previous 3 months. His medical history included diabetes for 1 year without treatment and smoking for 30 years, however, there was no family history of ischemic heart disease or other known coronary risk fac- 

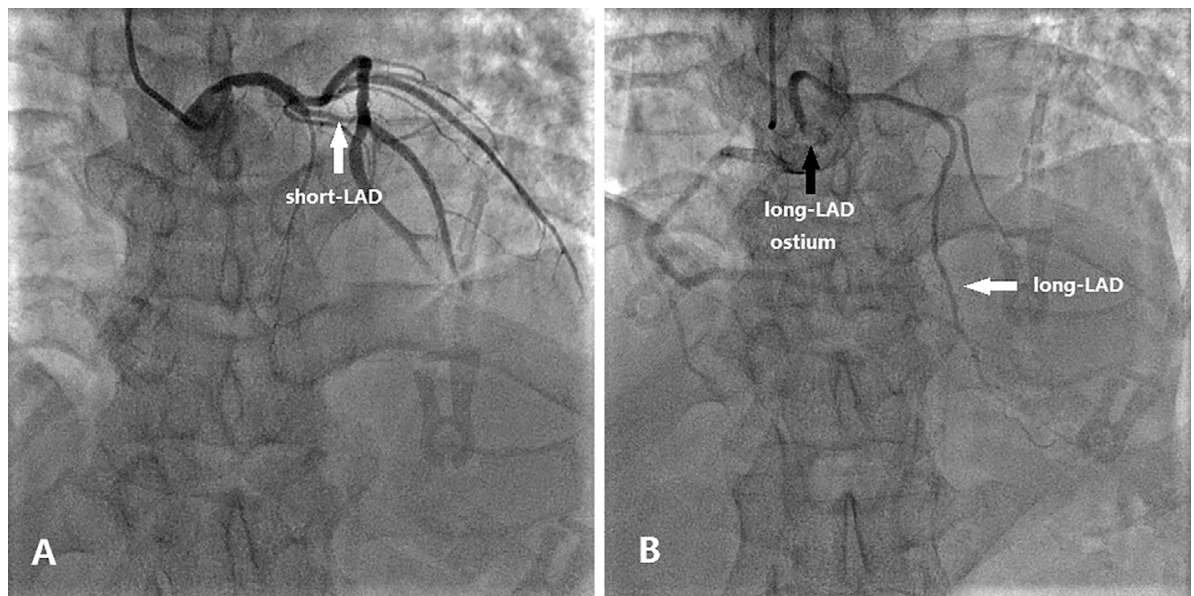

Figure 1. Coronal artery angiography: (A) Anteroposterior cranial view. The proximal short LAD has significant stenosis (white arrow). (B) Anteroposterior cranial view. The long LAD independently arises from the right coronary sinus of Valsalva (black arrow) and reaches to the anterior interventricular sulcus and ends at the apex (white arrow).

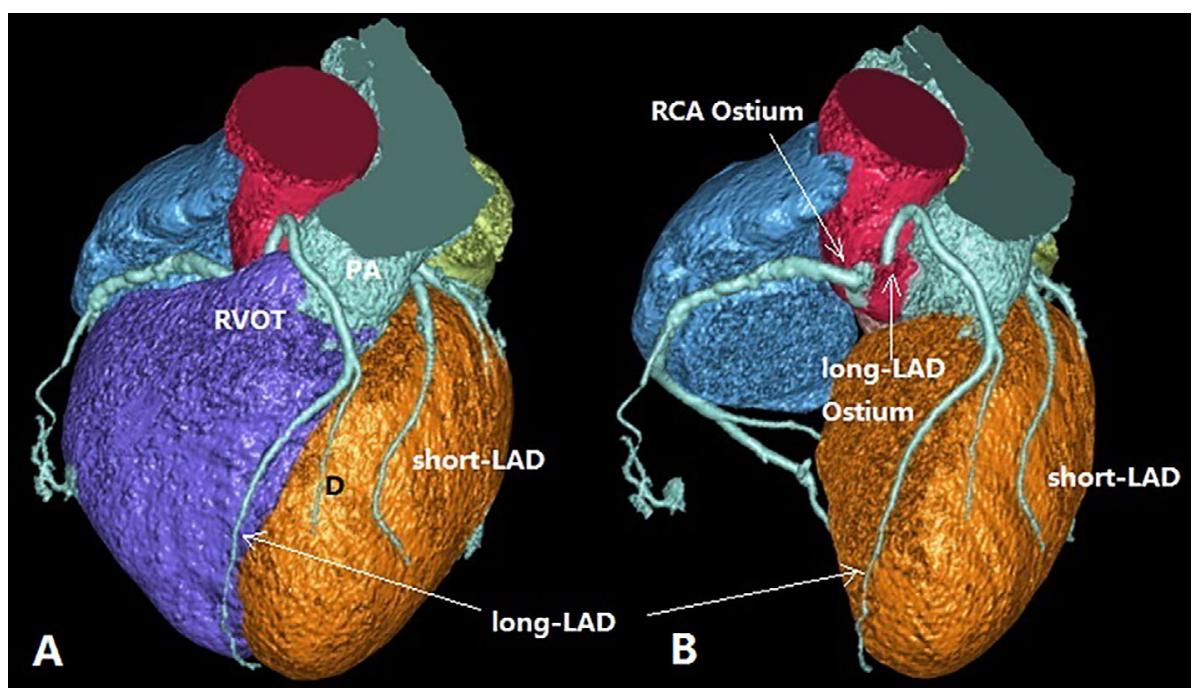

Figure 2. Three-dimension volume-rendered CT images of the coronary circulation: (A) Left anterior oblique cranial view, a reconstructed image of MDCT shows the long LAD course anterior to the right ventricular outflow tract (RVOT) and entering the mid anterior interventricular groove, where it gives rise to the diagonal branch. Finally, the long LAD arrives at the apex. (B) Left anterior oblique cranial view, with the RVOT removed, shows the long LAD arising from the right sinus of Valsalva independently. LAD: left anterior descending artery, RCA: right coronary artery

tors. On admission, the patient was asymptomatic. The physical examination was normal, and the electrocardiogram showed normal sinus rhythm without significant ST-segment changes. The left ventricular end diastolic diameter was 5.4 $\mathrm{cm}$, the left ventricular ejection fraction was 0.62 , and the coordinates of the left ventricular wall motion were determined by an ultrasound cardiogram. The levels of myocardial necrosis markers were normal. According to a detailed examination, stable angina was diagnosed and the patient was treated with peroral isosorbide mononitrate, aspirin, and statins.

Two days later, we performed coronary angiography, which revealed that the left circumflex artery and the short LAD arose from the LMCA (Fig. 1). The long LAD artery arose from the RSV. In the right anterior oblique projection, when the coronary artery formed the recent upward, which entered the anterior aspect of the interventricular septum where it provided a septal and diagonal branch and ended at the apex of heart. The left circumflex artery and the RCA were well developed.

To increase the understanding of this abnormal LAD and determine the spatial relationships between the long LAD and the surrounding structures, the patient underwent CT angiography (Fig. 2). It was found that the long LAD originated from the RSV, then entered the mid anterior interventricular groove, where it sent a diagonal branch. The long LAD ran into the ventricular crest and the septum. The CT angiogram showed the course of the LAD artery in the ante- 


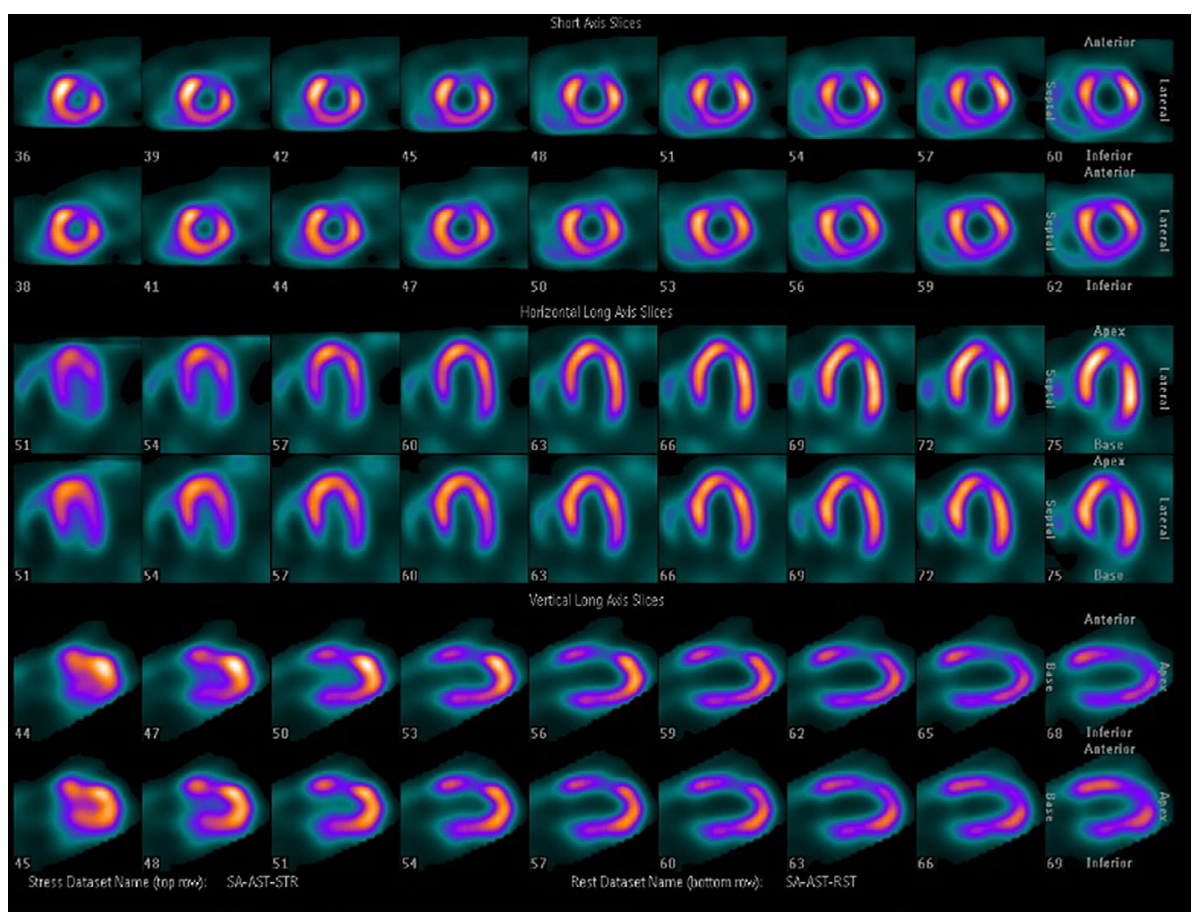

Figure 3. Single-photon emission-computed tomography (SPECT) in short axis, horizontal long axis, and vertical long axis standard views. (Top row) Stress images show left ventricular myocardial imaging clearly. Part of the left ventricular anterior wall and anteroseptal wall can be seen with detects. (Bottom row) The remaining images show that small parts of the above areas are partly filled, compared with the stress images.
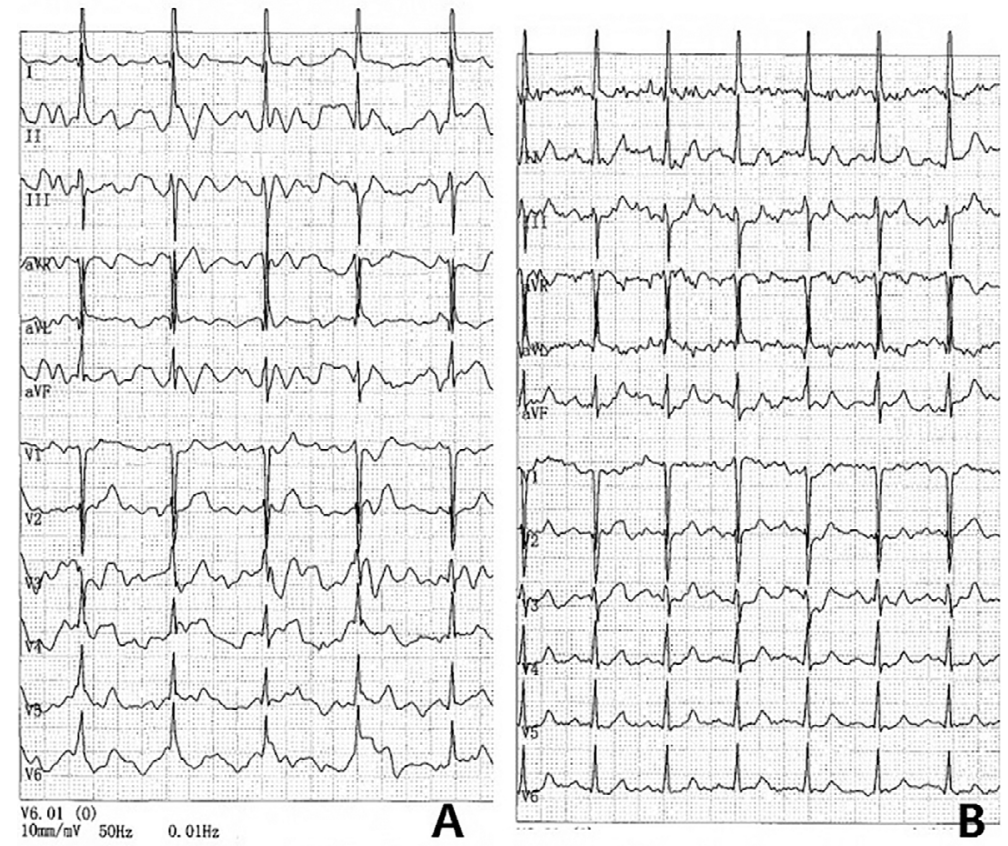

Figure 4. Electrocardiograms recorded during (A) rest and (B) exercise. The electrocardiogram recorded during exercise does not show significant ST-segment changes from the electrocardiogram recorded during rest.

rior pulmonary trunk and the anterior right ventricular outflow tract (RVOT). We then scheduled myocardial perfusion scintigraphy.

Single-photon emission computed tomography (SPECT) was performed with $20 \mathrm{mCi}$ of technetium-99m methoxyisobutylisonitrile after an exercise stress test and repeated with the patient at rest (Fig. 3). An electrocardiogram after exercise did not indicate significant ST-segment changes 
(Fig. 4). On the images taken after stress, hypoperfusion was seen in a part of the anterior wall and anteroseptal wall of the heart. Perfusion of these segments was less at rest, as shown on the images taken with the patient at rest. This indicates that most of the myocardial ischemia was persistent and most of the myocardial necrosis had already occurred. When combined with the normal levels of myocardial necrosis markers and no obvious dynamic electrocardiogram changes, these distributions and changes suggested an old anterior myocardial infarction.

The nearly normal long LAD and the myocardial ischemic area appeared to be mostly persistent during rest, indicating a limited viable myocardium. Although stenosis of the short LAD was significant, we did not perform a percutaneous coronary intervention but rather administered a secondary prevention for coronary heart disease. The patient was discharged without complications on the seventh day after admission and he has since experienced no further chest discomfort.

\section{Discussion}

Coronary artery anomalies are present at birth, but relatively few are symptomatic during childhood. Most anomalies are discovered as incidental findings during coronary arteriography or at autopsy. The incidence of coronary artery anomalies reported in various case series is between $0.6 \%$ and $1.5 \%$ on the coronal artery angiogram (5-8). A rare anatomic variant of LAD is described as dual LAD (i.e., dual anterior interventricular artery) which consists of two branches supplying the usual distribution of LAD in the left ventricle (1).

Eber et al. (9) reported 10 cases of double LAD in 3,000 consecutive coronary angiograms of adults. Aydinlar et al. (4) reported a prevalence of dual LAD of $0.041 \%$ in 12,059 patients undergoing coronary angiography. Type IV dual LAD is an extremely rare anomaly $(6,10,11)$, and an LAD originating from the bilateral sinus of Valsalva in type IV dual LAD is even rarer. In the present case, the long LAD originated from the RSV and entered the anterior interventricular sulcus and apex of heart, and the short LAD originated from the LMCA. Therefore, a diagnosis was made of type IV dual LAD, but specifically, the long LAD arose from the RSV.

There are several anomalous courses of the LAD possible from the RSV (12). The 4 most common can be classified as posterior, interarterial, anterior, or septal depending on the anatomic relationship of the anomalous vessel to the aorta and the pulmonary trunk. According to this classification, the MDCT and angiography findings in the present case are classified as an anterior or pre-pulmonary course.

Type IV dual LAD is subject to misdiagnosis and improper treatment when not recognized. Most of the LADs currently described in the literature are without severe stenosis, thus, the majority are given no treatment or medicine only. There has been only one previous patient who under- went percutaneous coronary intervention. Matsumoto et al. (13) reported that a 75-year-old man with diagnosed unstable angina received percutaneous coronary intervention for a short $\mathrm{LAD}$, and the case was later determined to be dual LAD by MDCT.

Coronary artery malformations are rarely identified during life and are typically first recognized at autopsy, largely because they are insufficiently considered and difficult to determine by the routine examination or clinical testing. It is also difficult to determine the course of an anomalous coronary artery using coronary angiography in some patients, especially when an anomalous artery courses between the great vessels or runs through the myocardium. MDCT is a powerful tool in assessing the spatial relationships between an anomalous coronary artery and surrounding structures and avoids unnecessary percutaneous coronary intervention (14-17). In the present case, MDCT was useful in determining the course of the long LAD. The reconstructed images, from which the RVOT was removed, showed that the long LAD passed the pre-pulmonary artery and ran an epicardial course to the anterior interventricular sulcus (Fig. 3).

Approximately $80 \%$ of the congenital anomalies of the coronary arteries are benign and clinically asymptomatic, while $20 \%$ are clinically significant (2). However, some patients may suffer from chest pain, syncope, heart failure, arrhythmias, and sudden death $(1,18,19)$. Acute myocardial infarction has also been reported (5). The prognostic factor in coronary artery anomalies is the pathway of the coronary artery arising from the aorta. Congenital anomalous coronary artery has been reported as the cause of $5-35 \%$ of sudden cardiac death in young people, because of dilation of the aortic root and pulmonary artery, which may lead to the compression of an abnormal coronary artery (18). Deaths from congenital anomalies of coronary arteries, particularly those that originate from the wrong aortic sinus and course between the aorta and pulmonary trunk, are found to be significantly associated with physical effort (18). Extravascular coronary compression can induce blood flow obstruction, which can in turn cause effort (stable) angina as well as syncope, and anomalous coronary arteries with an interarterial course are associated with sudden cardiac death. Abnormal LAD from the right sinus has been consistently associated with sudden death, with a course between the pulmonary artery and aorta (19). However, sudden death can also occur in cases in which the left coronary system originates from the right sinus, even when the anomalous artery does not pass between the great vessels (20).

In the absence of severe atherosclerosis, myocardial ischemia can occur if the left coronary artery arises from the right coronary artery and courses between the aorta and pulmonary artery (interarterial). The mechanism in this case is most likely the transient occlusion of the left coronary artery, caused by an increase in the blood flow through the aorta and the pulmonary artery, resulting in kinking or pinching of the artery (21). In the present case, the long 
LAD via the pre-pulmonary trunk did not induce significant ischemia.

In the present patient, it was interesting to note that the long LAD had an anterior pulmonary course, the entire coronary tree was free of lesion, and the short LAD had an apparent lesion. Considering the courses and myocardial blood flow range of the long and short LAD, we speculate that the myocardium ischemia was caused by the short LAD. Furthermore, the persistently significant and slightly reversible ischemia during exercise in SPECT may support a largely necrotic myocardium.

Although the majority of patients with an anomalous dual LAD are asymptomatic and do not show any complications, a therapeutic approach must be adapted for each patient. In asymptomatic cases, the age of the patient and the type of anomalous artery should be carefully evaluated before therapy. Surgical correction is generally appropriate for the patients who exhibit symptoms or when these anomalies are identified as severe in young patients. In the present patient, the patient was first administered intensive secondary preventive care because the course of the long LAD paralleled that of the pre-pulmonary artery and there was no significant stenosis, slight ischemia occurred after stress, and most of the cardiac muscle was already necrotic.

Written informed consent was obtained from the patient for the publication of this case report and accompanying images.

The authors state that they have no Conflict of Interest (COI).

\section{Acknowledgement}

We thank Ji Gang Yang who provided the single-photon emission computed tomographic original image and professional analysis. We thank Ting Ting Zhang who helped us complete the $3 \mathrm{D}$ reconstruction of the MDCT image.

\section{References}

1. Lee Y, Lim YH, Shin J, et al. A case report of type VI dual left anterior descending coronary artery anomaly presenting with nonST-segment elevation myocardial infarction. BMC Cardiovasc Disord 12: 101, 2012.

2. Spindola-Franco H, Grose R, Solomon N. Dual left anterior descending coronary artery: angiographic description of important variants and surgical implications. Am Heart J 105: 445-455, 1983.

3. Manchanda A, Qureshi A, Brofferio A, Go D, Shirani J. Novel variant of dual left anterior descending coronary artery. J Cardiovasc Comput Tomogr 4: 139-141, 2010.

4. Maroney J, Klein LW. Report of a new anomaly of the left ante- rior descending artery: type VI dual LAD. Catheter Cardiovasc Interv 80: 626-629, 2012.

5. Angelini P, Velasco JA, Flamm S. Coronary anomalies: incidence, pathophysiology, and clinical relevance. Circulation 105: 24492454, 2002.

6. Yamanaka O, Hobbs RE. Coronary artery anomalies in 126,595 patients undergoing coronary angiography. Cathet Cardiovasc Diagn 21: 28-40, 1990.

7. Garg N, Tewari S, Kapoor A, et al. Primary congenital anomalies of the coronary arteries: a coronary arteriographic study. Int J Cardiol 74: 39-46, 2000.

8. Aydinlar A, Cicek D, Seturk T, et al. Primary congenital anomalies of the coronary arteries: a coronary arteriographic study in Western Turkey. Int Heart J 46: 97-103, 2005.

9. Eber B, Kilzer K, Luha O, et al. Coronary artery anomalies in adulthood. Wien Med Wochenschr 141: 406-411, 1991.

10. Kosar F. An unusual case of double anterior descending artery originating from the left and right coronary arteries. Heart Vessels 21: 385-387, 2006.

11. Turhan H, Atak R, Erbay AR, Senen K, Yetkin E. Double left anterior descending coronary artery arising from the left and right coronary arteries: a rare congenital coronary artery anomaly. Heart Vessels 19: 196-198, 2004.

12. Ropers D, Gehling G, Pohle K, et al. Anomalous course of the left main or left anterior descending coronary artery originating from the right sinus of Valsalva: identification of four common variations by electron beam tomography. Circulation 105: e42-e43, 2002.

13. Matsumoto M, Yokoyama K, Yahagi T, et al. Double left anterior descending artery arising from right and left sinus of Valsalva in patient with acute coronary syndrome. Int J Cardiol 149: e40-e42, 2011.

14. Kunimoto S, Sato Y, Kunimasa $T$, et al. Double left anterior descending artery arising from the left and right coronary arteries: depiction at multidetector-row computed tomography. Int J Cardiol 132: e54-e56, 2009.

15. Kadiyala M, Beasley HS, Dua A. A rare case of anomalous left anterior descending artery with a large interarterial communication: an invasive and CT angiographic study. J Invasive Cardiol 19: e69-e72, 2007.

16. Budoff MJ, Ahmed V, Gul KM, Mao SS, Gopal A. Coronary anomalies by cardiac computed tomographic angiography. Clin Cardiol 29: 489-493, 2006.

17. Datta J, White CS, Gilkeson RC, et al. Anomalous coronary arteries in adults: depiction at multidetector row CT angiography. Radiology 235: 812-818, 2005.

18. Basso C, Corrado D, Thiene G. Congenital coronary artery anomalies as an important cause of sudden death in the young. Cardiol Rev 9: 312-317, 2001.

19. Virmani R, Burke AP, Farb A, et al. The pathology of sudden cardiac death in athletes. Cardiol Clin 15: 439-466, 1997.

20. Iskandar EG, Thompson PD. Exercise-related sudden death due to an unusual coronary artery anomaly. Med Sci Sports Exerc 36: 180-182, 2004.

21. Stougiannos $P$, Tousoulis D, Trikas A, et al. Anomalous origin and course of a dual left anterior descending coronary artery. Int $\mathbf{J}$ Cardiol 146: e53-e55, 2011.

(C) 2015 The Japanese Society of Internal Medicine http://www.naika.or.jp/imonline/index.html 